\title{
Fraud in clinical research
}

The recent report on data falsification in a study on high-dose chemotherapy that Werner Bezwoda presented in the 1999 ASCO meeting has generated a considerable, and justified, concern in the international scientific community. This lamentable occurrence provides a good opportunity to reflect on fraud in clinical research; a problem to which, in my opinion, there has not been paid sufficient attention on the part of researchers, scientific societies and editors of biomedical journals. What constitutes «fraud» in research? The latest definition by the North American of- fice of Research Integrity states this: «Research misconduct is plagiarism; fabrication or deliberate falsification of data, research procedures, or data analysis; or other deliberate misrepresentation in proposing, conducting, reporting, or reviewing research» ${ }^{1}$. Many of these irregularities of conduct were present in the Bezwoda case and which, without doubt, will become the paradigm of fraud in clinical research.

In case any reader is unaware of the details of the Bezwoda case, what follows is a brief description of the facts. At the annual meeting of the American Society of Clinical Oncology (ASCO), celebrated in May 1999 in Atlanta, doctor Bezwoda of the University of Witwatersrand (South Africa) presented the results of a randomized study comparing conventional CAF chemotherapy to high-dose CNVp (cyclophosphamide, mitoxantrone, vincristine) vith autologous haematopoietic support as adjuvant therapy in women with high-risk, operable breast cancer ${ }^{2}$. The women treated with high-dose chemotherapy had a disease-free survival and an overall survival significantly superior to that of the women treated with CAF. To corroborate the results of this provocative trial prior to initiating an international confirmatory study, a team of North American clinicians conducted an on-site audit of the patients' records and the documents related to the protocol ${ }^{3}$. The auditors had considerable trouble completing their assessments (for example, the clinical records of the patients that had

Rev Oncología 2001; 3: 57-59 been assigned to the standard treatment arm were not made available). They discovered numerous irregularities in the study. Outstanding among these irregularities were: evidences of manipulation of the written protocol that was provided to them, absence of approval ofthe study by the ethical committee of the institution, absence of written informed consent from the patients, inconsistencies between the data generated and that which were presented at the ASCO meeting, inclusion of a high proportion of patients that were not eligible with respect to the original protocol criteria, etc. Further, they encountered evidence that the patients in the control arm had, in reality, been treated with CNV instead of CAF; Dr. Bezwoda repeatedly denied this point when questioned by the auditing team.

In January 2000, the Committee for Investigation in Human Subjects of the University of Witwatersrand was alerted to the irregularities encountered and it solicited an explanation from Dr. Bezwoda. The investigator admitted his fraudulent activity and informed the committee that the standard treatment administered to the patients in the control arm had been CNV. He justified his conduct, in writing, with the following statement «This was done out of a foolish desire to make the presentation more acceptable to an audience who I believed would have regarded CAF as a more familiar and a more acceptable control arm». As a consequence of this scandal, the University of Witwatersrand dismissed the professor from his post in the institution ${ }^{4}$.

What have been the consequences of this fraud? Firstly, within the controversy on the usefulness of high-dose adjuvant chemotherapy in breast cancer, the results that Bezwoda communicated lent credit to the argument in favour of its routine use and, as such, many women in the world were subjected to a toxic treatment whose efficacy is, as yet, unproven. Secondly, when the fraud became known, many detractors of high-dose chemotherapy and most medical insurance companies exploited the situation to create a state of opinion that was totally negative to the therapy. As a 
consequence of this, a dramatic reduction occurred in the recruitment of patients into randomised studies that compared intensive chemotherapy with conventional chemotherapy in breast cancer and, as such, there is a well-founded fear that the true usefulness of this therapy will never be known. Thirdly, this fraud has brought into question the capacity of scientific organisations and biomedical journals to guaranty the truth of the data for whose dissemination they are responsible. The Journal of Clinical oncology had published in 1995 another randomised article by Bezwoda et al, in which high-dose chemotherapy was demonstrated to be clearly superior to the conventional chemotherapy in metastatic breast cancer ${ }^{5}$. In this study several inconsistencies were encountered between the text and the tables and which leads to high suspicion of fraud or, at least, of data manipulation.

On the positive side, the case of Bezwoda has induced an element of reflection within the international scientific community. Numerous doubts had arisen before the blow of the discovery of this unfortunate affair. Does fraud exist in a more-orless extensive manner in clinical research? Can we have confidence in the data in the literature? Is the prestige and meticulousness of an investigator, an institution or a work group sufficient to guaranty us the veracity of the data they present? Is the prestige of a journal sufficient to convince us of the veracity of the studies that it publishes? The Bezwoda case constitutes a fraud that we could categorise as being «major» (invention of data, non-existence of a formal proto$\mathrm{col}$, falsifying the description of the control therapy, etc.). Other examples of «major» fraud are the fabrication of non-existent results, plagiarism and the intentional manipulation of results of a study so as to obtain conclusions that are not sustainable by the original data. These lamentable aspects of conduct have been discovered on rare occasions over the last few years. Nevertheless, many of us are aware of other «lesser frauds» such as inconsistencies between the protocol and the actual conduct of the study, inclusion of ineligible patients, inadequate exclusion of eligible patients, deficient data collection and inadequate statistical analyses. These deficiencies are much more common in single-centre studies that are not subject to extemal quality control audits. In many cases, these manipulations can completely alter the conclusions of a clinical trial.

With the current requirements for publication of studies, which are based on a declaration by the authors regarding the veracity of data, the deficiencies mentioned above cannot be detected and the investigators are not put under any pressure to adopt measures of quality control of their data. It appears evident that the editors of biomedical journals or the organisers of scientific meetings should, when approving an article for publication or when selecting and abstract for an oral communication, assume a certain responsibility for verifying the validity of the data. However, the present system of review of manuscripts and abstracts is absolutely ineffective in detecting fraud. Arnold S. Relman, editor of the New England Journal of Medicine in the $80 \mathrm{~s}$, defined this ineffectiveness very well. Commenting on the case of Darsee, an investigator who published two articles based on fraudulent data in this prestigious journal, he posed the somewhat rhetorical question: «What kind of protection against fraud does peer review offer? Little or none» ${ }^{6}$. Some editors of medical journals argue that interventionist practices such as auditing the data of studies that are submitted for publication are not within the remit of an editor and propose, instead, the creation of some sort of Official Agency for this purpose. In the manner of a tribunal, the experts of this agency would convene in the case of suspected fraud'. Indeed, this type of agency has already been created in the United States, Denmark, Norway, Finland and Australia and can be put into effect any time a study is suspected of being fraudulently produced. However, an agency of this type cannot resolve the principal problem: the detection of fraud.

We feel that editors of medical journals should seriously consider implementing a systematic policy for fraud detection. Recently, a Committee on Publication Ethics (COPE) has been set up by medical editors to support each other in scientific publishing. We hope that this committee defines certain guidelines to prevent, detect and handle fraud at the editorial level. Similarly, congress organisers (especially the scientific organisations) need to derive some formula to control for the veracity of, at least, the principal communications. On their part, investigators need to acquire a culture of strict rigour in the management of data from their clinical studies. Cooperative groups, in particular, should establish a system of quality control of clinical data that goes through regular in situ audits. This system is already in place in some groups such as GEICAM (Grupo Español de Investigación en Cáncer de Mama); the North American National Cancer Institute (NCI) requires a similar control to American Cooperative groups for NCI-sponsorized studies ${ }^{8}$.

Hopefully there could be a gathering of clinical investigators, scientific societies and editors of journals to establish an international consensus for new «rules of the game» regarding the hand- 
ling of data and communication of results of clinical trials. The consensus would need to be more in keeping with the rigour and seriousness that clinical research demands today. Some suggestions for discussion to improve the current situation, in my opinion, are the following:

1. Clinical publications should include, within the Material and Methods section, a statement referring to the quality control of the data. This section should contain a description of the steps taken to guaranty the veracity of the study. These should include information on patient registration, procedures for the randomisation of patients, methods of data collection, quality control measures (including external auditing) and the identification of the individuals responsible for the data handling as well as the statistical analysis. If data recording and control are performed according to good clinical practice rules (i.e., GCP-IG), it should be specified as well. In this way the reader has clear reference points within which to gauge the reliability of the conclusions of the study.

2. At the time of submitting a manuscript for publication, the authors should place at the disposal of the editor all documents that support the correct realization and the results of the study. These should include a summary of the original study protocol, documents of approval from the institution's ethical committee, patient's informed consent document, model data registration forms and even the database of the study; the anonymity of the patients being guarantied.

3. The authors should agree to accept an in situ audit of the results and to facilitate its execution should it be required by the editor of the joumal. Since 1989, the journal JAMA has required that the following statement be signed by the investigators submitting manuscripts for publication: «If requested, I shall produce the data upon which this manuscript is based for examination by the editors or their assignees» ${ }^{9}$. Who should perform the audit is another question for debate.

The veracity of results of communications presented at congresses and scientific meetings is more difficult to control, essentially because of the high numbers and the speed with which a deci- sion needs to be taken whether to accept or reject an abstract. As has been clearly cited, fraud at conferences has been the object of very little attention to-date ${ }^{10}$. The incongruities of the data in the study of Bezwoda, for example, were not detected by the reviewers of the ASCO and ECCO-10 conferences (both being top ranking meetings); this has been considered an evidence of the inefficiency of the «abstract-review procedures»" Some if not many of the abstract that are accepted in quite prestigious scientific meetings are of very poor quality. The commercial interest of conference organisers could, in part, explain this lack of rigorous selection. Incidentally, the great number of studies that are presented at scientific meetings but which never reach the stage of publication in a medical journal is an aspect worth seriously reflecting upon. Hopefully a convenient and effective form of quality control of data in congress communications could be established, at least for those communications of major importance which are selected for oral presentation in the more important sessions of the meeting.

In the fight against fraud, as with the struggle against disease, prevention is the more effective intervention. We are convinced that implementing the measures mentioned above, or others that are similar, will exercise a dissuasive action on fraud in clinical research. Perhaps, at the same time, penalties (within certain well-defined legal limits) should be placed on authors who participate in fraudulent scientific activity or who reject extemal audit. This could take the form of extensive publicity in the joumals regarding the individual participants as well as the details of the fraud. Alternatively, it may be worthwhile to consider a ban or refusal to consider for publication, for a statutory period of, say, 5 years any article emanating from such authors.

Miguel Martín Servicio de Oncología Médica. Hospital Universitario San Carlos. Madrid. Chairman, GEICAM

\section{References}

1. Rennie D, Gunsalus CK. Scientific misconduct. New definition, procedures and office. Perhaps a new leaf. JAM A 1993; 269: 915-917. 
2. Bezwoda WR. Randomised, controlled trial of high dose chemotherapy (HD-CNVp) versus standard cose (CAF) chemotherapy for hifh risk, surgically treated, primary breast cancer. Proc ASCO 1999; 18: 2 (abstract 4) .

3. Weiss RB, Riflin RM, Stewart FM, et al. High dose chemotherapy for high-risk primary breast cancer: an onsite review of the Bezwoda study. Lancet 2000; 355: 999-1.003.

4. Wits fires cancer researcher: press release of the University of the Witwatersrand Medical School, Johannesburg, South Africa, March 10, 2000. Http://www.wits. ac.za/depts/wcs/media.

5. Bezwoda WR, Seymur L, Dansey RD. High dose chemotherapy with hematopoietic rescue as primary treatment for metastatic breast cancer: a randomised trial. J Clin Oncol 1985; 13: 2.483-2.489.

6. Relman AS. Lessons from the Darsee affair. N Engl J Med 1983; 308: 1.415-1.417.

7. Farthing MUG. An editor's response to fraudsters. British Med J 1998; 316: 1.713-1.729.

8. Weiss RB, Vogelzang HJ, Peterson BA, et al. A successful system of scientific data audits for clinical trials. A report from the cancer and leukemia Group B. JAMA 1993; 270: 459-464.

9. Rennie D. Accountability, audit and reverence for the publication process. JAMA 1993; 270: 495-196.

10. Rymer J. Fraud at conferences needs to be addressed. British Med J 1998; 317: 1.591.

11. Horton R. After Bezwoda. Lancet 2000; 355: $942-$ 943. 